\title{
Observations Illustrating the Use of Health Informatics to Link Public Health Immunization Registries and Pharmacies to Increase Adult Immunization Rates and Improve Population Health Outcomes
}

\author{
Michael Popovich, MSSE; Brandy Altstadter, BSISE; Lara Hargraves Popovich, BS Biology
}

Scientific Technologies Corporation

\begin{abstract}
The Health Information Technology for Economic and Clinical Health (HITECH) Act encourages health information exchange between clinical care and public health through Meaningful Use measures. Meaningful Use specifically identifies objectives to support a number of public health programs including immunizations, cancer registries, syndromic surveillance, and disease case reports. The objective is to improve public and population health. Stage 2 of Meaningful Use focused on compliance to sending of information to public health. The next phase focuses on bi-directional information exchange to support immunization intelligence and to empower providers, pharmacists, and the consumer.
\end{abstract}

The HITECH Act Stage 2 initiative provided incentive and motivation for healthcare providers to encourage their Electronic Medical Record (EMR) vendors to implement data exchanges with public health, with the expected result being timely awareness of health risks. The empowerment nugget in the HITECH Act is not in the compliance reporting to public health. The nugget is the ability for a provider to receive relevant information on the patient or consumer currently in front of them or to those they will connect to through their outreach efforts.

The ability for public health to retain current immunization records of individuals from a variety of providers supports their program goals to increase immunization rates and mitigate the risk of vaccine-preventable disease (VPD). The ability for providers to receive at the point of service more complete immunization histories integrated with decision support enhances their delivery of care, thereby reducing the risk of VPD to their patients. Indirectly payers benefit through healthcare cost savings and when the focus is expanded from a health model to a business model, there are significant return on investment (ROI) opportunities that exponentially increase the value of a bidirectional immunization data exchange. This paper will provide descriptions of case examples to demonstrate the value of electronic data exchanges when pharmacy immunization providers and public health work together.

Key Words: Adult Immunizations, Meaningful Use, Immunization Information System (IIS), Electronic Health Records (EHRs), Immunization Intelligence, vaccine-preventable disease (VPD), public health informatics, population health outcomes, retail pharmacist, healthcare provider, bi-directional data exchange, Advisory Committee on Immunization Practices (ACIP)

Correspondence:: Michael_Popovich@stchome.com 
DOI: $10.5210 /$ ojphi.v8i2.6398

Copyright @2016 the author(s)

This is an Open Access article. Authors own copyright of their articles appearing in the Online Journal of Public Health Informatics. Readers may copy articles without permission of the copyright owner(s), as long as the author and OJPHI are acknowledged in the copy and the copy is used for educational, not-for-profit purposes.

\section{Introduction}

Retail pharmacies are playing an increasingly important role in immunization practices in the U.S. Pharmacists are providing patients with convenient access to immunization services creating opportunities for traditional public health organizations to improve vaccine coverage rates particularly for at-risk and underserved adolescent and adult populations. Traditional healthcare providers are characteristically not seeing the adolescent and adult populations on a regular basis. Visits to the physician after school age are less oriented to preventative care and more to the treatment of illness. The visit focus is not on an immunization event. To minimize missed opportunities physicians must actively check immunization status of each patient in addition to addressing the primary visit purpose.

Pay for performance and patient and population health outcomes for medical reimbursement have created the need for health record data sharing, improved medical record systems, and registries that capture information from multiple communities of care. As the information systems and data exchanges are extended to a community of care that includes pharmacists, the opportunity to integrate with state public health immunization registries that add decision support expands the current Immunization Information Ecosystem (IIe) for increasing vaccine coverage rates of all ages.

In the U.S., immunization registries have been evolving for over two decades. The statewide immunization information system (IIS) has become the single resource for recording consolidated patient immunization events. Today individuals of all ages are receiving immunizations in non-traditional clinical settings including in pharmacies, at the workplace, in travel clinics, and outside their normal area of residence. As immunization information is recorded in medical record systems the data in many is being electronically sent to an IIS for consolidation. The IISs exist in all fifty U.S. states ${ }^{1}$ and are becoming an important component of public health programs in the Canadian provinces as well.

Retail-based pharmacist vaccinators have not been active users of the data in the IISs historically, although pharmacists in the U.S. are required by most states through policy to report the immunizations they provide to public health. Figure 1 summarizes pharmacy vaccine administration authority by state, illustrating the growing landscape for pharmacist immunizations. $^{2}$ 


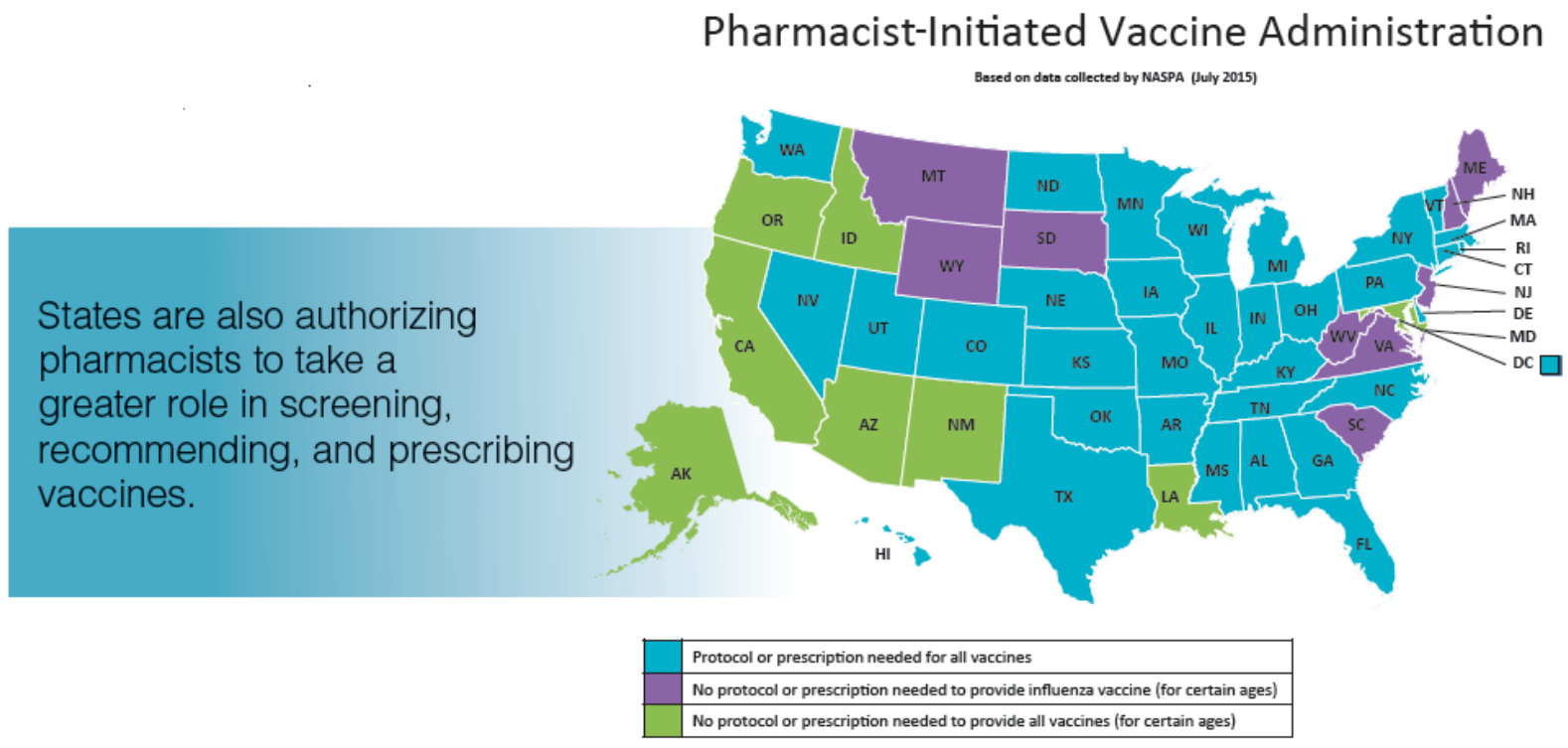

Figure 1. Summary by State Showing the Growing landscape for Pharmacist Immunization Administration

As a pioneer in developing and supporting immunization registries, Scientific Technologies Corporation (STC) began to partner with the pharmacy community during the 2009 U.S. H1N1 outbreak. This was the first real opportunity that empowered pharmacies to demonstrate their effectiveness in providing immunizations. Without their broad population reach, vaccinations for H1N1 influenza at that time would have been far less reaching and less effective.

It was also at this time that health policy began actively expanding to support making a full list of vaccinations available to the pharmacy. National chain and independent drugstores have begun to recognize a value-added component of their business practice as they implement immunization programs. The fact that a significant percent of the population shops regularly at retail stores that contain a pharmacy represents a major opportunity to improve population health through the immunization programs being offered. The American Pharmacists Association (APhA) has estimated that in a one-week span, 300 million individuals will shop in a retail setting that includes a pharmacy. At the April 2016 National Association of Chain Drug Stores (NACDS) annual conference, it was reported that in a given year an individual visits a pharmacy on average thirty times and a physician will visit a pharmacy on average twice.

The national chain drug stores and major retailers have been the first to recognize the value of an immunization program within their locations. Independent and community small chains have engaged equally in these programs to maintain their competitiveness and they all recognize the value-added potential for their customers. Given the momentum for pharmacy-based immunization programs, the value to public health is this new opportunity to reach populations, such as adults, outside of the traditional child and school-age individuals on which previous programs have focused.

STC has undertaken a series of evaluations in the past few years to determine the potential benefit of immunization programs to both the pharmacy and public health. This paper reviews 
lessons learned from three pharmacy case studies that provide real world applications of public health information technology in practice, with each demonstrating the power of the HITECH Act to return value and validate decisions to invest:

1) Five pharmacies of a small drugstore chain (with 65 stores) in Washington State which volunteered to evaluate the additional immunization opportunities when an individual presented at the pharmacy counter for a flu shot and at that time their records were retrieved from the state immunization registry.

2) A national retail chain with locations in all states illustrates technical and practice scalability.

3) A project that specifically targets adolescents and adults using registry data with added intelligence through decision support at the point of care.

\section{Literature Review}

In 2014, STC provided a White Paper "The Value of the State Immunization Registry towards Improving Vaccination Rates and Quality of Care in Retail Pharmacies, Achieving Patient-Centered, Quality Care through the Application of Immunization Registry Information when Combined with Best Practices," which detailed the Five Pharmacy Case Study summarized herein.

STC is planning an upcoming publication for the third quarter of 2016 focused on a new initiative on which STC is partnering with the Washington State Pharmacy Association, the Washington State Department of Health, and the American Pharmacists Association Foundation (APhA Foundation). This new study, with eight pharmacies participating, will conduct a formal study to measure and monitor the increase in additional immunizations during pharmacy flu clinics.

\section{Methods}

\section{Five-Pharmacy Case Study}

This study, conducted in early 2014 with Bartell Drugs, sought to identify the benefits of using the IIS from the pharmacist's perspective, and the potential that IIS information has on increasing the number of additional vaccinations administered at this point of care. As pharmacists are not traditional IIS users, a component of the study also was to evaluate the workflow impact and the use of information technology, and to identify barriers that could potentially inhibit their use of this data as part of their patient care.

In Washington State, pharmacists are allowed to both prescribe and administer vaccinations and are given access to the public health statewide IIS upon request. It has been estimated that approximately $35-40 \%$ of pharmacists used the state IIS in 2014, even though many have been administering vaccinations since 2011, with a number of pharmacists having vaccinated for as long as fifteen years. The question to answer was how this provider community can benefit from advances in the information and data environment. 
Little was known about whether patients would choose to receive additional vaccinations in this setting if they were informed of their immunization status and whether they were due or overdue for an immunization. Bartell pharmacists were making recommendations to patients based on their immunization histories which align with working toward a patient-centered approach to care, however, almost nothing was known about how pharmacists use the IIS or how vaccination information is shared with patients in practice.

The study involved five pharmacies and eight pharmacists as they expanded their immunization efforts to include consulting patient information contained within the Washington State IIS. This health data asset adheres to CDC immunization information system core standards ${ }^{3}$ and contained records of over 74 million immunization events for over eight million patients. The STC study team observed the pharmacists administering vaccinations to all patients arriving in the pharmacy who requested a vaccination. When a patient arrived for a vaccination, the pharmacist recorded the information into their existing pharmacy management record system as part of their normal patient enrollment process. The pharmacist would then augment their workflow separately by accessing the IIS to search for the patient's vaccination record.

The pharmacist retrieved and viewed vaccination historical events. A patient's vaccination forecast was available to the pharmacist based on the immunization records contained in the IIS. This forecast presented as a list indicating patient-specific vaccinations as either "Due" or "Overdue." The forecasting decision support tool used the Advisory Committee on Immunization Practices (ACIP) ${ }^{4}$ guidelines to forecast this information based upon age and the patient's previous immunization events. The pharmacist would use his or her own judgment to decide whether to recommend or offer any additional vaccinations to the patient. The patient had the opportunity to obtain one, all, or none of any recommended vaccinations. Observers recorded the vaccinations the pharmacist recommended and the vaccines the patient chose to receive.

Observers also recorded instances of when the pharmacist gave no recommendations and the reason for not recommending vaccines. If a patient refused recommended vaccinations, the refusal was recorded along with the reason for the patient's refusal. The entire process is diagrammed in Figure 2.

The most common overdue vaccinations were Varicella for Chickenpox and Measles, Mumps and Rubella (MMR). Pharmacists using the outcomes from the IIS decision support for the patient were most likely to recommend Meningococcal (66.7\%), Pneumococcal (50.0\%), Hep A $(50.0 \%)$ and Tdap $(50.0 \%)$ if those vaccinations were shown as overdue.

There was a strong association between the pharmacist and whether or not the patient chose an additional needed vaccination. This could indicate that patient choice was influenced by their pharmacist, with certain pharmacists more likely to elicit a patient choice of opting for additional vaccination. This small 2014 study showed a need for developing a best practices model and providing training on how to make the appropriate immunization recommendations. It also demonstrated that significant opportunity was missed to vaccinate individuals and thus reduce the risk of disease. Observers noted the impact on the pharmacist's time when this process was outside of their normal workflow. 


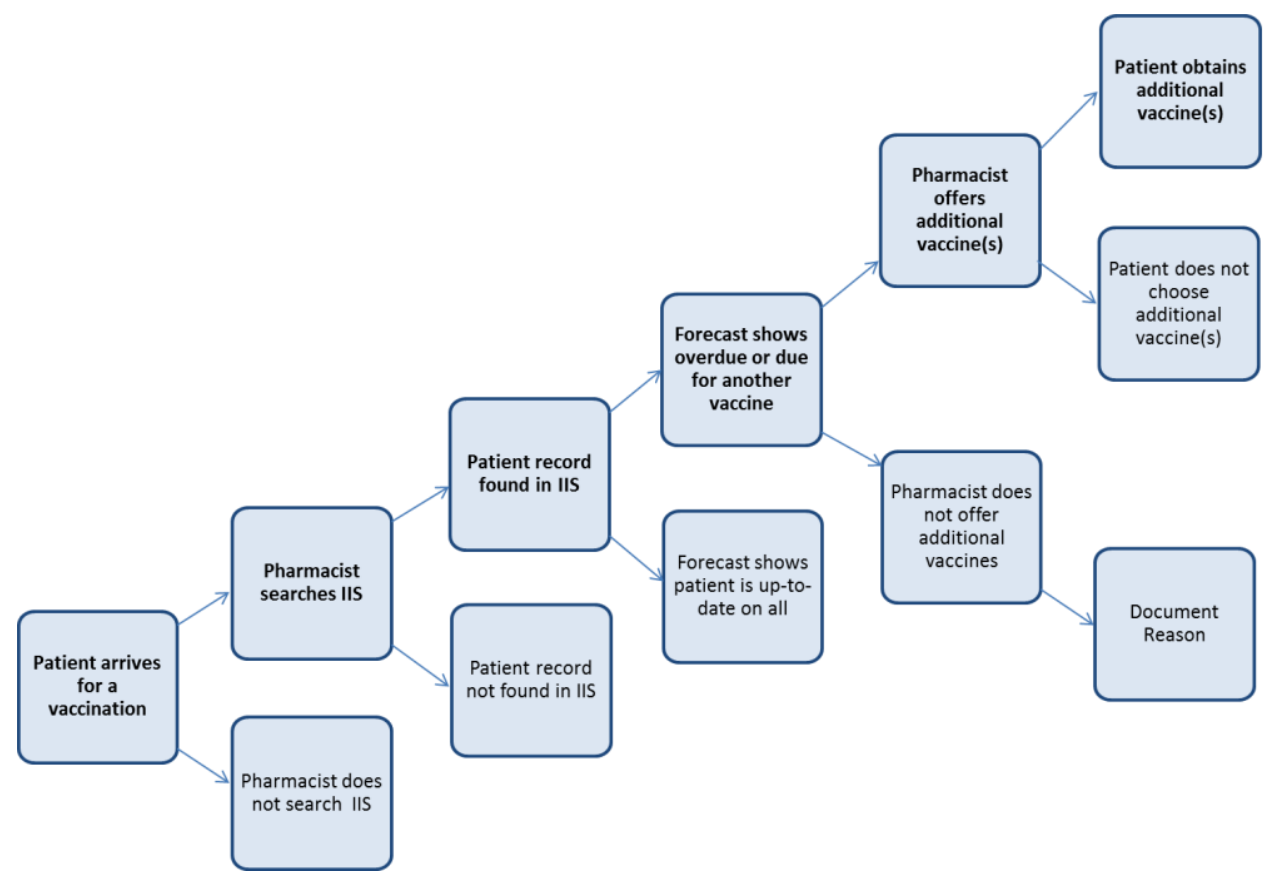

Figure 2. Pharmacist registry use, recommendation, and vaccination decision tree.

The overall results of the five pharmacies case study are shown in Table 1 .

Table 1. Results from each step in the pharmacist registry use, recommendation, and vaccination decision tree.

\begin{tabular}{|l|l|}
\hline $\begin{array}{l}\text { Proportion } \\
\text { of Total }\end{array}$ & Step in Registry Use and Recommendation Decision-Tree \\
\hline $100 \%$ & Patients arriving for a vaccination. \\
\hline $95 \%$ & Patients searched in the IIS. \\
\hline $74 \%$ & Patients found in the IIS. \\
\hline $62 \%$ & Patients due or overdue identified by the IIS vaccination forecast. \\
\hline 3.22 & Average vaccinations due or overdue per patient. \\
\hline $33 \%$ & Patients receiving a direct vaccination offer by the pharmacist. \\
\hline $5 \%$ & Patients that opted-in for additional vaccination. \\
\hline
\end{tabular}

Although not significant it was evident that within a pharmacy if the pharmacy management system (PMS) was interoperable with the IIS and through a single-sign on process the patient's records were available immediately upon entry in to the PMS the interaction with the patient would likely increase. It was also clear that this interoperability should include a bi-directional capability with decision support thereby allowing the immunization(s) given by the pharmacist's to be electronically sent to the IIS in real time and a Forecast to be presented at the point of care. 
And finally a preliminary understanding of the opportunity for added revenue was identified as it was noted "money was walking out the door." How much money was being lost? The Washington State IIS reported that 449,495 unique patients received a vaccination at a pharmacy between June 2013 and May 2014. Using the 3.22 average identified in this study for immunizations past due per individual, this represents 1,447,374 missed opportunities to vaccinate. To illustrate further the value to a pharmacy and the potential business: if the profit on providing each of these immunizations was $\$ 15$ each, it would represent a total of more than \$21 million in additional revenue for all Washington State pharmacies.

Advances in data availability, decision support, and pharmacy clinical practice was shown to be valuable to this small drugstore chain, to the point that the following year the chain implemented a complete system with electronic data exchange between the state IIS and all pharmacies and integrated decision support combined with training of pharmacists to message this information towards the goal of providing more recommended vaccinations to patients.

\section{Major National Chain Example}

The Washington State study was an early investigation that demonstrated the potential for significant ROI if the process was easily integrated into the daily workflow of the pharmacist. In 2015, STC began to work with a major U.S. retail business with thousands of pharmacies throughout the U.S. The initial effort entailed reporting compliance specifically to ensure that all retail pharmacies were able to electronically report the immunizations provided in each location to the appropriate state or local major metropolitan IIS. These links were secure HL7 data exchanges.

As the electronic exchange links were established a number of public health registries were able to support two-way data exchanges. Bi-directional exchanges, unlike the above Washington State example which required the pharmacist to look up a patient record on a separate terminal, offered the advantage of the single sign-on process and interoperable IIS connectivity. Secure HL7 message traffic from the IIS was able to return both the patient immunization events as contained in the public health dataset as well as add the decision support information that was determined automatically by the immunization forecaster.

As part of the project rollout, the patient match rate and completeness of the state IIS records were evaluated in two states. Both states require bi-directional connectivity per state law. The first question was to determine the likelihood that the pharmacy demographic would be found in the IIS. The typical pharmacy demographic in this retail setting includes over $50 \%$ of patients that are 65 or older (indicated for pneumococcal); between 10-15\% that are ages 60 to $64 ; 30 \%$ between the ages of 27 and 59; with the remainder (5-10\%) under 27 years old.

Both states reviewed have well-established lifetime immunization information systems. Data showed that the pharmacy patients were found in the IIS, on average, $73 \%$ of the time (77\% in the first state and $69 \%$ in the second) which was comparable to the Washington State example from the 2014 study.

The second question looked at the completeness of the IIS records. The graph in Figure 3 shows the average number of immunizations returned by age group. 


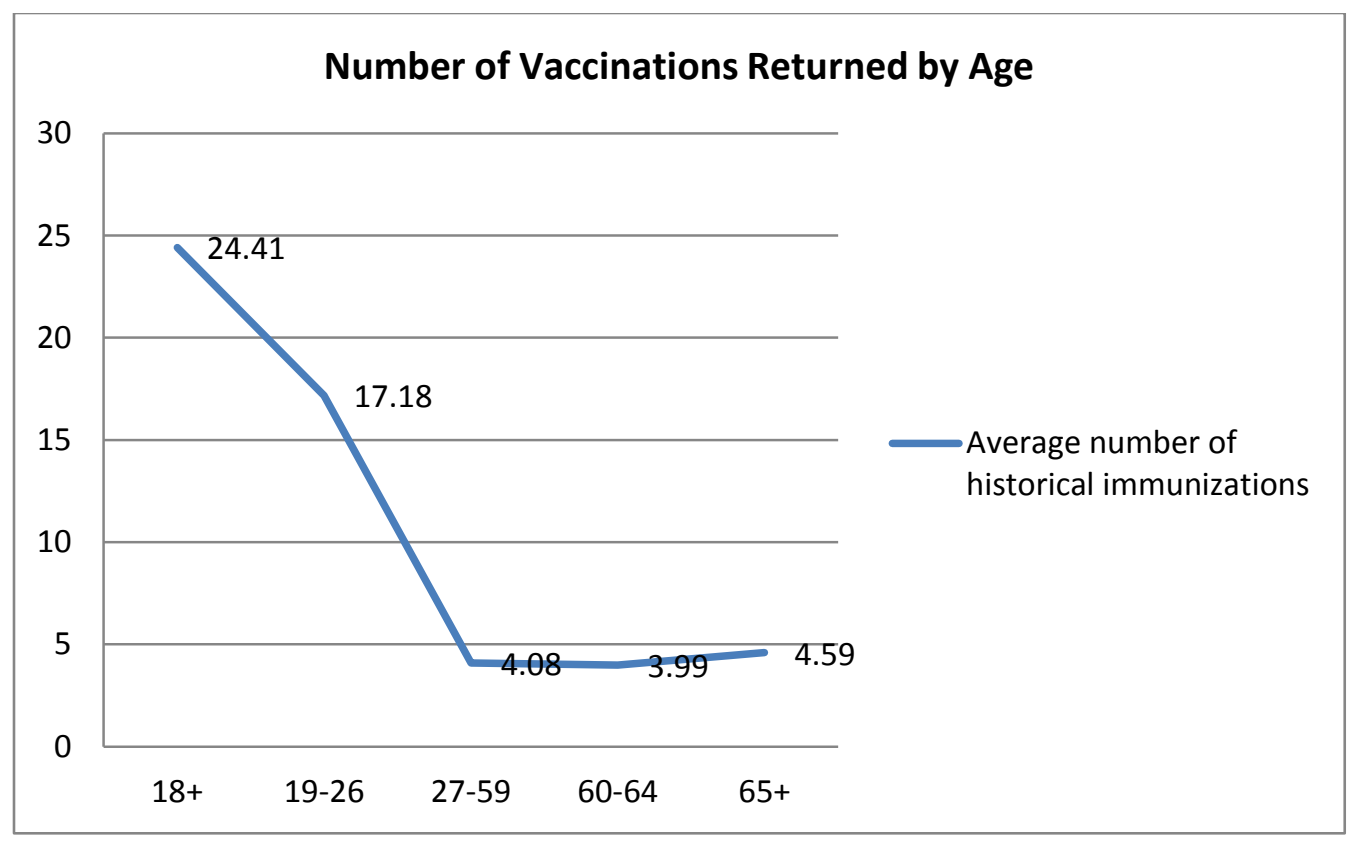

Figure 3. Number of vaccinations returned by age.

Of the patients searched and found in the IIS, 91\% were due for at least one additional vaccination. The third key question asked was, of the patients due for vaccinations, how many vaccinations were due for each age group. Table 2 details the results.

Table 2. Vaccinations due by age group.

\begin{tabular}{|llll|} 
Age & $\begin{array}{l}\% \text { due for one (1) } \\
\text { vaccination }\end{array}$ & $\begin{array}{l}\% \text { due for two (2) } \\
\text { vaccinations }\end{array}$ & $\begin{array}{l}\% \text { due for three (3) } \\
\text { or more vaccinations }\end{array}$ \\
\hline Under 18 & $13 \%$ & $22 \%$ & $65 \%$ \\
\hline $\mathbf{1 9 - 2 6}$ & $34 \%$ & $36 \%$ & $30 \%$ \\
\hline $\mathbf{2 7 - 5 9}$ & $94 \%$ & $5 \%$ & $1 \%$ \\
\hline $\mathbf{6 0 - 6 4}$ & $46 \%$ & $53 \%$ & $1 \%$ \\
\hline $\mathbf{6 5 +}$ & $19 \%$ & $37 \%$ & $45 \%$ \\
\hline
\end{tabular}

The above analysis, also presented at the American Immunization Registry Association (AIRA) 2015 National Meeting, shows a wealth of opportunity for pharmacies to improve adult patient outcomes by identifying patient immunization needs and addressing health needs that otherwise go unserved. A tightly integrated and continuously operating system for viewing immunization history and recommendations results in the opportunity for the pharmacist to engage the patient, at every visit, in a conversation about receiving needed vaccinations. 


\section{A Targeted Pharmacy Study}

As the ROI begins to illustrate the value to retail pharmacies, there is also the value of how pharmacists can play a critical role in increasing the uptake of vaccinations in high-risk populations or hard to reach populations. Adolescents and adults are commonly underimmunized after their initial childhood series that carried them through their school years. The 2014-2015 five-store pharmacy study which showed an average of 3.22 vaccinations due or overdue per patient suggested that specific public health programs could be implemented in partnership with local pharmacists to increase the uptake of targeted vaccines. This 2015 retail case example moved the manual workflow to an integrated automatic process which empowers pharmacists by eliminating barriers associated with added time for a manual process. Similar ROI was demonstrated in each case.

This case, a partnership of a state pharmacy association, a number of retail pharmacies, and state public health established an immunization-specific target. The results will be fully evaluated with expected publishing in mid-2016. The project is designed specifically to leverage technology, data exchange, and point of care immunizations at pharmacies to increase specific coverages rates in adults that are under-immunized. The pharmacists are actively engaging with the patient after the forecast is returned to proactively offer the missing immunizations.

Preliminary results from this project indicate that in a six-week period of time, the eight pharmacies provided flu vaccinations to just fewer than 1,000 individuals. Of these, over $78 \%$ had an immunization record in the state IIS. In this six-week period an additional 300+ immunizations were provided to those requesting a flu shot. When an immunization record was not found in the state IIS the pharmacist used age-appropriate calculations to suggest vaccinations that might be due or past due.

The secondary endpoints of this study include how often a patient declined to receive an additional immunization beyond flu and the reasons why which will help to identify gaps in both patient and pharmacist education. For example, early results have indicated that patients, at a rate of approximately 30\%, have declined both Pneumovax and Prevnar13. They cite the reason as they believe they have already received it.

This indicates a major gap in patient knowledge since the Prevnar13 vaccine is only two years old and should therefore have a lower coverage rate and overall higher documentation rate then its counterpart, Pneumovax. This data could prove to be invaluable to vaccine manufacturers when they are developing marketing campaigns directed to the consumer and creating provider educational resources.

\section{Discussion}

These three case studies show early indication of opportunities and potential benefits of establishing interoperable information systems with public health registries.

Anecdotal feedback from more than one pharmacist indicated that their ability to view the full patient immunization history and to be provided with the information that clearly illustrates what the individual needs has empowered them to provide facts at the point of care. Bi-directional 
exchange of information increased immunization intelligence for the pharmacist and the patient. Immunization intelligence created opportunity to increase vaccine coverage rates in at-risk populations. As a result of the initiatives through the HITECH Act we should expect population health outcomes that show measurable improvement when private-public partnerships work together. It is more than merely the sending and receiving of data - it is developing program alliances that also include common objectives, consumer education, and measurable performance indicators.

\section{Conclusion}

The HITECH Act created a technology roadmap that supports the objective of improving health outcomes by empowering patients and providers with timely and more complete health information. The foresight of supporting Meaningful Use objectives including public health reporting creates new opportunities to impact population health. Adding pharmacies whose immunization programs are exponentially expanding and pharmacists who are delivering increased numbers of immunizations to a broad range of patients into this environment and it is feasible to assess the value of electronic data exchange between all parties.

These studies involved early pharmacy adopters supporting public health immunization reporting and the use of information. In each case applied practice illustrated significant benefits to the patients, the pharmacy, and to public health. The ability to accelerate these connections and to develop messaging and consumer outreach will have a favorable impact on population health. Public health will be instrumental in building these public-private partnerships with electronic data exchanges forming the backbone of the effort.

\section{Limitations}

The case study examples and this article did not detail the technical frameworks of each case study, the standards used, or the integration into the workflow. All are important to understand applied health informatics in data exchanges and to increase the understanding of the costs to implement and support them. They are the topics of a series of follow-up papers in development by STC staff and partners to include:

(1) Technical Frameworks in an Interoperable Immunization Information Ecosystem, EMRs, Pharmacy Management Systems and Consumers. (Expected August 2016.)

(2) Demonstrated Impact on Adult Immunization Rates within Pharmacy Immunization Programs in Partnership with Public Health Immunization Information Exchanges (Expected October 2016.)

(3) The State of Data Exchanges with State Immunization Information Systems (First Quarter 2017.)

The case study examples did not address the data quality or the impact on the pharmacists' workflow nor did they evaluate the economic models and benefits although numerous observations provided for the next steps. 


\section{References}

1. As of May 26, 2016, New Hampshire became the final state in the U.S. to implement an IIS. The IIS is implemented and supported by STC.

http://www.dhhs.nh.gov/media/pr/2016/05262016registry.htm

2. http://www.pharmacist.com/pharmacist-administered-immunizations-what-does-your-stateallow

3. http://www.cdc.gov/vaccines/programs/iis/core-data-elements.html

4. http://www.cdc.gov/vaccines/schedules/hcp/index.html 